\title{
Coronal Mass Ejections travel time
}

\section{Carlos Roberto Braga, Rafael Rodrigues Souza de Mendonça, Alisson Dal Lago and Ezequiel Echer}

\author{
National Institute for Space Research, \\ Av. Dos Astronautas 1758, Jd. Granja, São José dos Campos, SP, Brazil \\ email: carlos.braga@inpe.br
}

\begin{abstract}
Coronal mass ejections (CMEs) are the main source of intense geomagnetic storms when they are earthward directed. Studying their travel time is a key-point to understand when the disturbance will be observed at Earth. In this work, we study the CME that originated the interplanetary disturbance observed on 2013/10/02. According to the observations, the CME that caused the interplanetary disturbance was ejected on 2013/09/29. We obtained the CME speed and estimate of the time of arrival at the Lagrangian Point L1 using the concept of expansion speed. We found that observed and estimated times of arrival of the shock differ between 2 and 23 hours depending on method used to estimate the radial speed.
\end{abstract}

Keywords. coronal mass ejections (CMEs), halo CMEs, interplanetary disturbances, magnetic clouds

\section{Introduction}

Coronal mass ejections (CMEs) and their corresponding structures in the interplanetary medium (known as ICMEs) are of great importance because they cause most of the intense geomagnetic storms (Gosling et al. 1990; Gonzalez et al. 1990). Those events are frequently called geoeffective CMEs when they have direction of propagation close to the Sun-Earth line (Möstl et al. 2014).

In this work we identify the CME that caused the ICME observed on 2013/10/03 and then estimate its travel time using only the speed extracted from coronagraph observations.

\section{Identifying the CME and estimating its travel time}

In order to identify the CME associated to the interplanetary disturbance, we used simultaneous observation from three white light coronagraphs onboard at three different spacecraft: the twin STEREO (Solar TErrestrial RElations Observatory, Kaiser et al. 2008) spacecraft and SOHO (Solar and Heliospheric Observatory, Domingo et al. 1995). While the later is located in the Lagrangian point L1, the STEREO probes were located approximately $1 \mathrm{AU}$ away from the Sun, one ahead of the Earth orbit by $147^{\circ}$ and the other behind by $139^{\circ}$ during the ICME observation period. Their separation angle was approximately $73^{\circ}$.

First, we inspected white-light coronagraph observations from the LASCO -C3 (Brueckner et al. 1995) onboard SOHO looking for identification of any CME possibly directed toward the Earth. Those CMEs are seen as halos or partial halos surrounding the occulting disk. From previous studies, we know that the travel time of the CME ranges basically from 1 to 5 days (Gopalswamy et al. 2001, Schween et al. 2005). From our analysis, the unique CME observed as a partial halo on the period was first seen on the LASCO coronagraph C2 at 2013/09/29 22:12. No full halo event was identified. 

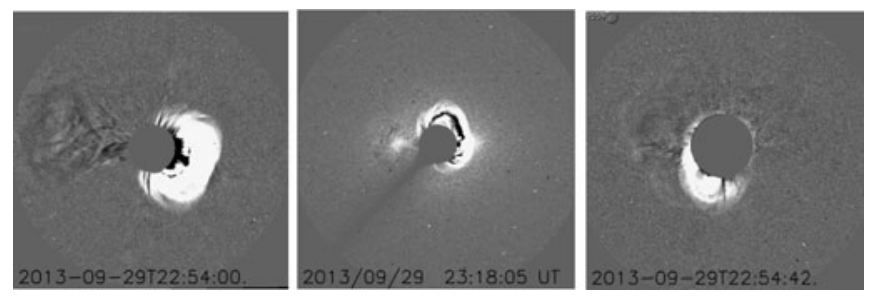

Figure 1. The observation of the CME on 2013/09/29 on both COR2 A (left), COR2B (right) and LASCO -C3 FOVs.

Secondly, we identified the corresponding CME observed on the COR2 coronagraphs onboard the STEREO spacecraft. Due to its viewpoint, a CME directed toward the Earth is observed as a eastward limb on COR2A and as a westward limb on COR2B. From this analysis we confirm that the CME observed on 2013/09/29 22:12 is directed toward the Earth and must be the cause of the ICME (Fig. 1).

According to the in situ observation from the instruments MAG and SWEPAM onboard the ACE spacecraft (located at the Lagrangian Point L1), an interplanetary shock was observed on 2013/10/02 01:18, more than 50 hours after the first observation of the $\mathrm{CME}$ on the solar corona. The solar wind increased to approximately $600 \mathrm{~km} / \mathrm{s}$ and the interplanetary magnetic field reached values of $32 \mathrm{nT}$. On the first half of 2013/10/03, a structure with magnetic cloud (MC) signatures was observed, in accordance with the criteria from Lepping et al. (2005)

The definition of the CME time of arrival (ToA) at L1 will depend on the specific signature observed in situ interplanetary data and there is no consensus about the parameter to be used (see, e.g., Gopalswamy et al. 2003). Beyond the time of the shock observation, other parameters used are: (a) the peak magnetic field intensity associated to the shock (2013/10/02 04:24); (b) the beginning time of the MC (2013/10/02 22:35) and (c) peak density (2013/10/02 03:58).

The ToA can be estimated using the expansion speed, a speed perpendicular to the radial direction of the CME (Dal Lago et al. 2003). The CME travel time $T_{\text {travel }}$ can be estimated by an optimum fit function (from Schwenn et al. 2005): $T_{\text {travel }}=203-$ $20.77 \ln \left(v_{\text {exp }}\right)$ where $T_{\text {travel }}$ is given in hours and $v_{\text {exp }}$ is the expansion speed (given in $\mathrm{km} / \mathrm{s}$ ). Using the pseudo-automatic segmentation of the CME from CORSET methodology, we derived $v_{\text {exp }}=837 \mathrm{~km} / \mathrm{s}$ (the reader is referred to Braga et al. 2013 for details). In this case the travel time estimated is 63 hours and the ToA is 2013/10/02 13:25. When we do not have the expansion speed, it can be derived from from the fit between the $v_{\text {exp }}$ and $v_{\text {rad }}$ obtained by Dal Lago et al. (2004): $v_{\text {exp }}=v_{\text {rad }} / 0.88$.

We took the radial speed derived by CME catalogs such as the CDAW CME catalog (Yashiro et al. 2004 ), CACTus (Computed Aided CME Track, Robbrecht \& Berghmans 2004) and SEEDS (Solar Eruptive Event Detection System, Olmedo et al. 2008). By estimating the expansion speed from the radial speed, we calculated the ToA. In the specific case of CORSET, the ToA was calculated in two ways: (i) using the expansion speed provided from the method ignoring the radial speed and (ii) ignoring the expansion speed and using the radial speed to estimate it.

Among all methods cited in the upper paragraph, the ToA estimated differs from the shock observation time the least when using the radial speed from CDAW catalog. In this case, the estimated ToA is 2013/10/02 03:39, approximately 2 hours later than the observed shock. When using the results from CORSET, the difference increases to approximately half a day: 2013/10/02 13:25 using the expansion speed, and 2013/10/02 
12:29 using the radial speed to estimate the expansion speed. The speeds from fully automatic catalogs (CACTus and SEEDS) resulted in estimated ToA 20 hours later than the shock (2013/10/03 04:17 and 2013/10/03 00:04, respectively). Notice that these two ToAs lie in the first hours of the MC-like observation. One possible explanation for the best ToA estimation obtained when using the speed derived by CDAW catalog is the similarity between the methodology used in this catalog and the one adopted by Schween et al. (2005) and Dal Lago et al. (2004): both methodologies identify the CME by eye. On the other methodologies, the CME speed is derived using automatic or pseudo-automatic identification.

\section{Final remarks}

The ToA of the CME observed on 2013/09/29 was estimated using the methodology from Schween et al. (2005) and taking plane-of-sky projected speed measurements from LASCO-C3 derived using several methods. For all radial speeds considered here, the estimated ToA was found to be latter than the actual shock observation and the difference ranges from 2 to 23 hours, depending on the method used.

\section{Acknowledgments}

C. R. Braga acknowledges grants \#2014/24711-6, \#2013/02712-8 and \#2012/05436-9 from São Paulo Research Foundation (FAPESP). A. Dal Lago acknowledges CNPq for grant 304209/2014-7. E. Echer acknowledges CNPq for grant 302583/2015-7. R. R. S. de Mendonça acknowledges and CNPq for grant 152050/2016-7. LASCO is one of a complement of instruments on the Solar Heliospheric Observatory satellite (SOHO) built in an international collaboration between ESA and NASA. The Sun Earth Connection Coronal and Heliospheric Investigation (SECCHI) was produced by an international consortium and it is part of the STEREO spacecraft.

\section{References}

Braga, C. R., Dal Lago, A., \& Stenborg, G. 2013, ASR, 51,1949

Brueckner et al. 1995, Solar Phys., 162, 357

A. Dal Lago, Schwenn, R. \& Gonzalez, W. D. 2003, ASR, 32, 2637

Domingo, V., Fleck, B., \& Poland, A. I. 1995, Solar Phys., 162, 1

Gonzalez, W. D., Tsurutani, B. T., \& Clúa de Gonzalez, A. L. C. 1999, SSRv, 88, 529.

Gopalswany, N., Lara, A., Yashiro, S. et al. 2001, JGRA, 106, 12, 29207

Gopalswamy, N., Manoharan, P. K., \& Yashiro, S. 2003, GRL, 30, 2232

Gosling, J. T. 1990, in: Russell, C. T. , Priest, E. R. \& Lee,L. C. Physics of Magnetic Flux Ropes, Geophysics Monograph, 58 (Washington, DC: American Geophysical Union), 343

Kaiser, M. L., Kucera, T. A., Davila, J. M. et al. 2008, SSRv, 136, 5

Lepping, R. P., Wu, C. C., \& Berdichevsky, D. B. 2005, AnGeo, 23, 2687

Liu, Y. D., Luhmann, J. G., Möstl, C. et al. 2012 ApJ (Letters), 746, L15

Möstl, C., Amla, K., Hall, J. R. et al. 2014, ApJ, 787, 119

Robbrecht, E. \& Berghmans, D. 2004, A\& A, 425, 1097

Schwenn, R., Dal Lago, A., Huttenen, E., \& Gonzalez, W. D. 2005, AnGeo, 23, 1033

Yashiro, S., Gopalswamy, N., Michalek, G. et al. 2004, JGRA, 109, A07105 